\title{
Determinants for changing the treatment of COPD: a regression analysis from a clinical audit
}

\author{
This article was published in the following Dove Press journal: \\ International Journal of COPD \\ 2 June 2016 \\ Number of times this article has been viewed
}

Jose Luis López-Campos, ${ }^{1,2}$ María Abad Arranz,' Carmen Calero Acuña, ${ }^{1,2}$ Fernando Romero Valero, ${ }^{3}$ Ruth Ayerbe García, ${ }^{4}$ Antonio Hidalgo Molina, ${ }^{3}$ Ricardo I Aguilar Perez-Grovas, ${ }^{4}$ Francisco García Gil, ${ }^{5}$ Francisco Casas Maldonado, ${ }^{6}$ Laura Caballero Ballesteros, ${ }^{5}$ María Sánchez Palop, ${ }^{6}$ Dolores PérezTejero, ${ }^{7}$ Alejandro Segado, ${ }^{7}$ Jose Calvo Bonachera, ${ }^{8}$ Bárbara Hernández Sierra, ${ }^{8}$ Adolfo Doménech, ${ }^{9}$ Macarena Arroyo Varela, ${ }^{9}$ Francisco González Vargas, ${ }^{10}$ Juan J Cruz Rueda ${ }^{10}$

'Unidad Médico-Quirúrgica de Enfermedades Respiratorias, Instituto de Biomedicina de Sevilla (IBiS), Hospital Universitario Virgen del Rocío/Universidad de Sevilla, Seville, ${ }^{2}$ Centro de Investigación Biomédica en Red de Enfermedades Respiratorias (CIBERES), Instituto de Salud Carlos III, Madrid, ${ }^{3}$ Hospital Universitario Puerta del Mar, Cádiz, ${ }^{4}$ Hospital Juan Ramón Jiménez, Huelva, ${ }^{5}$ Hospital Universitario Reina Sofía, Córdoba, ${ }^{6}$ Hospital Universitario San Cecilio, Granada, ${ }^{7}$ Hospital Infanta Margarita, Cabra, Córdoba, ${ }^{8} \mathrm{Hospital}$ Torrecárdenas, Almería, ${ }^{9}$ Hospital Regional Universitario de Málaga, ${ }^{10}$ Hospital Universitario Virgen de las Nieves, Granada, Spain

Correspondence: Jose Luis López-Campos Unidad Médico-Quirúrgica de Enfermedades Respiratorias, Instituto de Biomedicina de Sevilla (IBiS), Hospital Universitario Virgen del Rocío/Universidad de Sevilla, Avda. Manuel Siurot, s/n, 41013, Seville, Spain

Email Icampos@separ.es
Introduction: This study is an analysis of a pilot COPD clinical audit that evaluated adherence to guidelines for patients with COPD in a stable disease phase during a routine visit in specialized secondary care outpatient clinics in order to identify the variables associated with the decision to step-up or step-down pharmacological treatment.

Methods: This study was a pilot clinical audit performed at hospital outpatient respiratory clinics in the region of Andalusia, Spain (eight provinces with over eight million inhabitants), in which $20 \%$ of centers in the area (catchment population 3,143,086 inhabitants) were invited to participate. Treatment changes were evaluated in terms of the number of prescribed medications and were classified as step-up, step-down, or no change. Three backward stepwise binominal multivariate logistic regression analyses were conducted to evaluate variables associated with stepping up, stepping down, and inhaled corticosteroids discontinuation.

Results: The present analysis evaluated 565 clinical records (91\%) of the complete audit. Of those records, 366 (64.8\%) cases saw no change in pharmacological treatment, while 99 patients (17.5\%) had an increase in the number of drugs, 55 (9.7\%) had a decrease in the number of drugs, and $45(8.0 \%)$ noted a change to other medication for a similar therapeutic scheme. Exacerbations were the main factor in stepping up treatment, as were the symptoms themselves. In contrast, rather than symptoms, doctors used forced expiratory volume in 1 second and previous treatment with long-term antibiotics or inhaled corticosteroids as the key determinants to stepping down treatment.

Conclusion: The majority of doctors did not change the prescription. When changes were made, a number of related factors were noted. Future trials must evaluate whether these therapeutic changes impact clinically relevant outcomes at follow-up.

Keywords: quality of care, outpatient care, treatment strategies, follow-up, respiratory diseases, airway diseases

\section{Introduction}

Over recent years, the traditional concept of COPD as a constantly progressing disease has been challenged. Recent publications have shown that the clinical expression and functional impairment have an important component of variability. ${ }^{1,2}$ In addition, new treatment guidelines are proposing new diagnostic and therapeutic schemes based on different combined variables, multidimensional indices, or clinical phenotypes. ${ }^{3-5}$ Consequently, in clinical practice, the pharmacological treatment of COPD frequently must be adjusted between follow-up visits.

Interestingly, in recent decades, the publications and number of clinical guidelines regarding COPD have been continuously increasing. ${ }^{6,7}$ As one of the most common respiratory conditions, most regional, national, and international respiratory scientific societies have developed their own clinical guidelines for COPD or adopted an 
international one. However, the implementation of these guidelines in clinical practice is far from optimal. ${ }^{8,9}$ One common feature of these guidelines is that they are generally quite specific in defining how to start drug treatment. However, the guidelines are vague when defining how to modify treatment based on changes in the clinical expression of the disease, its progression over time, or in consideration of the recommendations.

Some of the controversies include the reinforcement with double bronchodilation, ${ }^{10}$ the introduction or discontinuation of inhaled corticosteroids (ICS), ${ }^{11}$ the use of different oral treatments such as preventive antibiotic therapies or phosphodiesterase 4 inhibitors, ${ }^{12,13}$ or the possibility to stepdown therapies. ${ }^{14}$ However, clinical practice guidelines are not as clear in recommending when to step-up or step-down treatment in different clinical scenarios. Consequently, the decision regarding when to step-up or step-down treatment in clinical practice is left to the clinician in charge. Unfortunately, the data indicating which variables clinicians should use to make these decisions remain unclear.

In Spain, a recent pilot COPD clinical audit evaluated the adherence to guidelines for patients with COPD in a stable disease phase during a routine visit in specialized secondary care outpatient clinics. ${ }^{15}$ The present study aimed to evaluate the information recorded in this audit to analyze prescribed treatment in a routine follow-up visit of COPD. In particular, we sought to identify cases with treatment changes and to analyze which variables were associated with the decision to step-up or step-down treatment.

\section{Methods}

This study was a pilot clinical audit performed in hospital outpatient respiratory clinics in the region of Andalusia, Spain (eight provinces with over eight million inhabitants). The methodology has been extensively previously reported. ${ }^{15}$ Briefly, $20 \%$ of centers in the area were invited to participate in this audit. Center selection was based on their participation in previous audits and on a voluntary basis. As a pilot study, randomization was not performed; therefore, we did not aim to achieve a representative sampling.

Cases with an established diagnosis of COPD based on risk factors, clinical symptoms, and a post-bronchodilator forced expiratory volume in 1 second $\left(\mathrm{FEV}_{1}\right) /$ forced vital capacity (FVC) ratio of $<0.70$ were deemed eligible. ${ }^{3}$ Our goal was to assess the utility of formally scheduled regular follow-up visits; therefore, only cases with at least 1 year of follow-up were included in the audit. Patients who underwent a first diagnostic visit or presented with an exacerbation were not eligible. Similarly, subjects with significant respiratory comorbidities that could impact the COPD treatment approach at the local investigator's discretion were also excluded.

As of 2008, our team has been leading clinical audits in Spain and Europe. ${ }^{16,17}$ Based on our previous experience, we estimated that 80 cases per center would be required for this pilot study. The 1-year audit occurred between October 2013 and September 2014. Recruitment was performed during four separate 3-month periods (October-December 2013, January-March 2014, April-June 2014, and July-September 2014). At the beginning of each period, investigators were instructed to identify consecutive COPD cases at the beginning of each trimester until the desired sample size of 20 per trimester was reached.

During the clinical visit that was audited, information regarding the actual treatment the patients were receiving was recorded, noting the name of the drug and the pharmacological family. The names of drugs ultimately prescribed after the audited visit were also noted. Changes were evaluated in terms of the number of drugs prescribed as a step-up, step-down, or no change. Treatments were categorized as long-acting $\beta_{2}$ agonists (LABA), long-acting muscarinic antagonists (LAMA), ICS, and fixed-dose combinations (FDCs) of an ICS and a LABA. Fixed combinations of longacting bronchodilators (LAMA+LABA) in one single inhaler were not available in the country at the time of the audit.

The Ethics Committee of the Hospital Universitario Virgen del Rocío approved the audit (code: 2013PI/201). Clinical records were deidentified in the database by assigning a numerical code through an algorithm. No personal information was registered that could be directly or indirectly used to identify an individual. The relationship between the audit code and the clinical history number was kept locally under the local investigator's supervision. Because of the retrospective nature of the study, the deidentification of data, and the lack of active research interventions, the need for informed consent was waived.

\section{Statistical analyses}

All analyses were performed using the Statistical Package for Social Sciences, version 20.0 (SPSS 20.0; IBM Corporation, Armonk, NY, USA). Clinical variables are presented as the mean and standard deviation or absolute and relative frequencies in parentheses, as appropriate. The variability between centers was expressed using the interhospital range, which represents the highest and lowest mean value from the participating centers. The significance of this variability 
was explored using the chi-square test or analysis of variance (ANOVA) test between the different participant centers. We then explored those variables associated with stepping up or down treatment, as well as those associated with ICS discontinuation, using an unpaired Student's $t$-test (after applying Levene's test of the equality of variances) or chisquare test, depending on the nature of the variable. Finally, those significant variables were modeled into a backward stepwise binomial multivariate logistic regression analysis. We built three models for stepping up, stepping down, and ICS discontinuation. The results are expressed as odds ratios (OR) with $95 \%$ confidence intervals (CIs). The alpha error was set at 0.05 .

\section{Results}

The audit evaluated 621 clinical records. In 56 of these cases $(9.0 \%)$, a clear decision on treatment change was unavailable. Thus, the sample size for the present study was 565 cases. The characteristics of the audited cases are summarized in Table 1 . These patients were mostly males in the seventh decade of life, with a considerable proportion of current smokers, a homogeneous distribution of comorbidities, and moderate-to-severe lung function impairment.

In 366 cases $(64.8 \%)$, there was no change in the pharmacological treatment, while 99 cases (17.5\%) showed an increase in the number of drugs and 55 cases (9.7\%) had a decrease. In 45 cases $(8.0 \%)$, there was a change to a similar therapeutic scheme using other drugs. Cases with no treatment change were receiving LABA (75 cases [20.5\%]), LAMA (321 cases [87.7\%]), FDC (241 cases [65.8\%]), ICS (20 cases [5.5\%]), roflumilast (44 cases [12.0\%]), mucolytics
(33 cases [9.0\%]), long-term antibiotics (eight cases $[2.2 \%]$ ), and theophylline (ten cases [2.7\%]).

Table 2 summarizes the primary changes observed. The most common step-up in treatment was to add a long-acting bronchodilator to the regimen in 58 cases (58.5\%), followed by adding an ICS in 19 cases (19.1\%). The most common step-down strategy was ICS discontinuation in 33 cases $(60.0 \%)$. Among those with a change to a similar scheme, the most common change was the change of the LAMA in 25 cases $(55.6 \%)$.

\section{Step-up associations}

The bivariate associations of factors related to treatment step-up are summarized in Table S1. For instance, the hospital itself was associated with a step-up in treatment. Three centers had an increased probability of stepping up treatment, including center 7 (15.9\% no step-up vs $7.1 \%$ step-up; $P=0.026)$, center 8 (7.3\% no step-up vs $2.0 \%$ step-up; $P=0.067)$, and center 9 (12.4\% no step-up vs $23.2 \%$ step-up; $P=0.011)$. The rest of the comorbidities evaluated were not significant between step-up and non-step-up patients. The time of year was also not related to stepping up. Multivariate determinants of stepping up are summarized in Table 3. Both FDC salmeterol-fluticasone and budesonide-formoterol were identical in their association with a risk of stepping up (data not shown). Two centers were associated with stepping up but with an opposite net effect.

\section{Step-down associations}

The bivariate associations of factors related to treatment step-down are summarized in Table S2. Neither the specific

Table I Clinical characteristics of the audited cases $(n=565)$

\begin{tabular}{|c|c|c|c|}
\hline Variable & Average $^{a}$ & Interhospital range & $P$-value ${ }^{b}$ \\
\hline Age (years) & $68.6(9.7)$ & $66.9-69.8$ & NS \\
\hline Male sex (n) & $480(85.0)$ & $47.4-94.8$ & $<0.001$ \\
\hline Current smokers (n) & $|3|(23.2)$ & $16.3-36.1$ & $<0.001$ \\
\hline Tobacco history (pack-year) & $55.2(31.1)$ & $42.5-65.7$ & $<0.001$ \\
\hline Comorbidities (Charlson) & $2.1(1.5)$ & $1.7-2.4$ & NS \\
\hline Psychiatric comorbidities (n) & $116(20.5)$ & I2.3-87.7 & NS \\
\hline Cardiovascular comorbidities (n) & I5I (26.7) & $18.2-38.9$ & NS \\
\hline Previous neoplasms ( $\mathrm{n}$ ) & $80(14.2)$ & $5.3-21.5$ & NS \\
\hline Time from diagnosis (years) & $5.6(6.1)$ & $1.1-6.9$ & $<0.001$ \\
\hline Exacerbations in the previous year $(n)$ & $0.9(1.3)$ & $0.5-1.3$ & NS \\
\hline Hospitalizations in the previous year ( $n$ ) & $0.2(0.6)$ & $0.04-0.37$ & $<0.001$ \\
\hline Body mass index $\left(\mathrm{kg} / \mathrm{m}^{2}\right)$ & $28.2(5.3)$ & $27.7-29.6$ & 0.007 \\
\hline FVC (\%) & $75.3(20.8)$ & $63.7-104.7$ & 0.001 \\
\hline FEV & $52.5(19.6)$ & $26.3-59.1$ & 0.017 \\
\hline
\end{tabular}

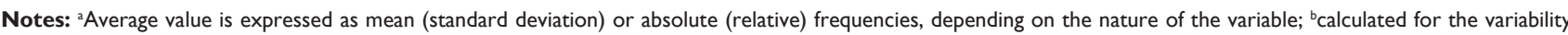
between centers using ANOVA or chi-square test, depending on the nature of the variable.

Abbreviations: FVC, forced vital capacity; $\mathrm{FEV}_{1}$, forced expiratory volume in I second; NS, not significant; ANOVA, analysis of variance. 
Table 2 Description of treatment changes

\begin{tabular}{|c|c|c|c|c|c|}
\hline Treatment step-up (n=99) & & Treatment step-down $(n=55)$ & & Treatment change $(n=45)$ & \\
\hline LAMA added: & $32(32.4)$ & LAMA discontinuation: & $10(18.2)$ & Change of LAMA & $25(55.6)$ \\
\hline LAMA added & $29(29.3)$ & & & Change of LAMA for LABA & $2(4.4)$ \\
\hline LAMA-LABA added & $3(3.0)$ & & & & \\
\hline LABA added: & $26(26.1)$ & LABA discontinuation: & $5(9.1)$ & Change of LABA for LAMA & $2(4.4)$ \\
\hline LABA added & $14(14.1)$ & LABA discontinuation & $3(5.5)$ & & \\
\hline LAMA-LABA added & $3(3.0)$ & FDC discontinuation & $6(10.9)$ & & \\
\hline ICS change for FDC & $2(2.0)$ & Change FDC for LAMA & $3(5.5)$ & & \\
\hline LAMA change for FDC & $3(3.0)$ & & & & \\
\hline FDC added & $4(4.0)$ & & & & \\
\hline ICS added: & $19(19.1)$ & ICS discontinuation: & $33(60.0)$ & Change of FDC & $7(15.6)$ \\
\hline ICS added & $5(5.1)$ & ICS discontinuation & $24(43.6)$ & Change of FDC for LABA-LAMA & $4(8.9)$ \\
\hline Change LABA for FDC & $7(7.1)$ & FDC discontinuation & $6(10.9)$ & & \\
\hline Change LAMA for FDC & $3(3.0)$ & Change FDC for LAMA & $3(5.5)$ & & \\
\hline FDC added & $4(4.0)$ & & & & \\
\hline Oral treatments added: & & Oral treatments discontinuation & & Change theophylline for roflumilast & $2(4.4)$ \\
\hline Roflumilast added & $17(\mid 7.1)$ & Roflumilast discontinuation & $10(18.2)$ & Other changes & $8(17.8)$ \\
\hline Mucolytic added & $8(8.1)$ & Mucolytic discontinuation & $3(5.5)$ & & \\
\hline Antibiotic added & $7(7.1)$ & Antibiotic discontinuation & $2(3.6)$ & & \\
\hline Theophylline added & $6(6.1)$ & Theophylline discontinuation & $8(14.5)$ & & \\
\hline
\end{tabular}

Note: Values expressed as absolute (relative) frequencies.

Abbreviations: LAMA, long-acting muscarinic antagonists; LABA, long-acting $\beta_{2}$ agonist; ICS, inhaled corticosteroids; FDC, fixed-dose combination of an inhaled steroid and a long-acting $\beta_{2}$ agonist.

treatment center nor the time of year was significantly associated with stepping down treatment.

Multivariate determinants of stepping up are summarized in Table 4. The main determinants of stepping down were being treated with long-term antibiotics, ICS, and the degree of lung function impairment as determined by $\mathrm{FEV}_{1}$.

\section{ICS discontinuation}

The bivariate associations of factors related to treatment step-up are summarized in Table S3. For instance, center 6 had an increased probability of ICS discontinuation $(P=0.055)$. There was no difference across the different trimesters.

Multivariate determinants of stepping up are summarized in Table 5. Center 6 remained in the model as being significantly associated with ICS discontinuation. Additionally, symptomatic Global lnitiative for Obstructive Lung Disease (GOLD) patient types B and D and a more severe lung function impairment also had a lower chance of ICS discontinuation. Investigators were more confident in discontinuing ICS when the patient was receiving double bronchodilator therapy.

\section{Discussion}

This study examined the prescribed treatment at a routine follow-up visit for COPD treatment, analyzing the determinants of treatment change. The results indicate that the majority of doctors did not change the treatment regimen. Among those whose therapeutic regimen changed, disease exacerbation was the main drive to step treatment up,

Table 3 Multivariate analysis indicating variables associated with an increased probability of stepping up treatment

\begin{tabular}{|c|c|c|c|c|}
\hline \multirow[t]{2}{*}{ Variable } & \multicolumn{2}{|c|}{ Crude } & \multicolumn{2}{|c|}{ Adjusted } \\
\hline & OR & $95 \% \mathrm{Cl}$ & OR & $95 \% \mathrm{Cl}$ \\
\hline Center 8 & 0.262 & $0.062-1.109$ & 0.184 & $0.039-0.871$ \\
\hline Center 9 & 2.129 & $1.239-3.659$ & 1.864 & $1.017-3.417$ \\
\hline Acute myocardial infarction & 1.757 & $0.933-3.307$ & 1.968 & $0.969-3.994$ \\
\hline Chronic bronchitis & $\mathrm{I} .874$ & $1.202-2.922$ & $\mathrm{I} .824$ & $1.108-3.001$ \\
\hline Exacerbations I-2/year & 2.233 & $1.344-3.710$ & 2.696 & $1.544-4.707$ \\
\hline Exacerbations 3 or more/year & 3.484 & $1.718-7.064$ & 5.235 & $2.280-12.018$ \\
\hline Tiotropium & 0.343 & $0.220-0.535$ & 0.368 & $0.228-0.595$ \\
\hline ICS-LABA combination & 0.353 & $0.227-0.550$ & 0.260 & $0.156-0.431$ \\
\hline
\end{tabular}

Abbreviations: OR, odds ratio; $\mathrm{Cl}$, confidence interval; ICS, inhaled corticosteroids; LABA, long-acting $\beta_{2}$ agonist. 
Table 4 Multivariate analysis indicating variables associated with an increased probability of stepping down treatment

\begin{tabular}{|c|c|c|c|c|}
\hline \multirow[t]{2}{*}{ Variable } & \multicolumn{2}{|c|}{ Crude } & \multicolumn{2}{|c|}{ Adjusted } \\
\hline & $\overline{\text { OR }}$ & $95 \% \mathrm{Cl}$ & $\overline{O R}$ & $95 \% \mathrm{Cl}$ \\
\hline Receiving antibiotics & 4.366 & I.299-14.678 & 15.909 & $2.812-90.025$ \\
\hline Receiving any ICS & 3.906 & $1.640-9.302$ & 4.287 & $1.351-13.598$ \\
\hline $\mathrm{FEV}_{1}(\%)$ & 1.021 & $1.002-1.040$ & 1.033 & $1.011-1.055$ \\
\hline
\end{tabular}

Abbreviations: $\mathrm{OR}$, odds ratio; $\mathrm{Cl}$, confidence interval; ICS, inhaled corticosteroids; $\mathrm{FEV}_{\text {, }}$, forced expiratory volume in I second.

while long-term antibiotics and ICS were the preferred drugs to discontinue. More specifically, symptomatic and severely impaired patients as well as those with asthma-like symptoms, together with the use of two long-acting bronchodilators were associated with ICS discontinuation. Previous observational studies have described the prescription profile in different clinical settings and geographic areas. ${ }^{918-20}$ These studies described the prescription patterns; however, the present analysis indicates what type of change the doctors made and the determinants of these changes.

Clinical audits are conceived as tools to summarize the clinical performance of health care over a specified period of time and aimed at providing information to health professionals to allow them to assess and adjust their performance. ${ }^{21}$ In practical terms, health professionals can receive feedback on their performance based on data derived from their routine practice. While it appears intuitive that health care professionals would be prompted to modify their clinical practice in response to feedback that their practice was inconsistent with that of their peers or accepted guidelines, such modification can be influenced by several other factors. ${ }^{22}$ Accordingly, an audit should be followed by not only feedback but also an implementation program. Although clinical performance has been exhaustively studied during admissions, situations in outpatient clinics have only been evaluated in one preliminary report in Italy. ${ }^{23}$

The main strength of this study is the novel information it provides and the wide coverage in catchment population screened at a regional level. However, when interpreting our results, a number of issues must be considered. First, the participating hospitals volunteered, and there was no attempt at representative sampling. As a consequence, it is possible that the hospitals involved in the study were those with an improved performance, which clearly constitutes a selection bias. Therefore, this pilot study should be used to estimate sample size calculation for a major randomized nationwide clinical audit. Second, another limitation intrinsically associated with audits is the considerable number of investigators who recorded the information using different information sources. The auditors were clinicians capable of diagnosing and treating COPD patients. The advantage is that the auditors are familiar with the disease and are thus qualified to correctly evaluate the clinical records and the information recorded; however, they are not expert auditors. Therefore, we provided specific instructions on how to perform the audit and record the information. Third, this audit focused on a cross-sectional evaluation of one particular clinical visit and is therefore cross-sectional in design. It is important to evaluate the impact of guideline adherence on clinically relevant outcomes. Accordingly, a follow-up of patients is needed to evaluate exacerbation rates and survival.

The observed relevance of the different centers to changes in treatment is worth noting further. The current guidelines

Table 5 Multivariate analysis indicating variables associated with an increased probability of discontinuing inhaled steroids

\begin{tabular}{|c|c|c|c|c|}
\hline \multirow[t]{2}{*}{ Variables } & \multicolumn{2}{|c|}{ Crude } & \multicolumn{2}{|c|}{ Adjusted } \\
\hline & OR & $95 \% \mathrm{Cl}$ & OR & $95 \% \mathrm{Cl}$ \\
\hline Center 6 & 2.205 & $1.011-4.813$ & 6.030 & $1.931-18.835$ \\
\hline GOLD A & 2.265 & $0.974-5.267$ & 1.667 & $0.584-4.818$ \\
\hline GOLD B & 0.401 & $0.088-1.814$ & 0.134 & $0.020-0.891$ \\
\hline GOLD C & 0.664 & $0.250-1.763$ & 0.342 & $0.100-1.167$ \\
\hline GOLD D & 0.250 & $0.07 I-0.878$ & 0.126 & $0.026-0.611$ \\
\hline Asthma-like symptoms & 0.364 & $0.108-1.235$ & 0.243 & $0.060-0.982$ \\
\hline Pneumococcal vaccine & 0.230 & $0.05 \mathrm{I}-\mathrm{I} .040$ & 0.191 & $0.035-1.034$ \\
\hline Not combined LABA+LAMA & 3.045 & I. $143-8.114$ & 5.753 & $1.717-19.277$ \\
\hline Mild FEV, impairment & $\mathrm{I} .333$ & $0.254-7.007$ & 0.452 & $0.063-3.260$ \\
\hline Moderate FEV, impairment & 0.197 & $0.050-0.768$ & 0.110 & $0.022-0.563$ \\
\hline Severe FEV, impairment & 0.211 & $0.058-0.770$ & 0.174 & $0.034-0.881$ \\
\hline Very severe FEV, impairment & 0.063 & $0.007-0.600$ & 0.050 & $0.004-0.640$ \\
\hline
\end{tabular}

Abbreviations: OR, odds ratio; $\mathrm{Cl}$, confidence interval; LAMA, long-acting muscarinic antagonists; LABA, long-acting $\beta_{2}$ agonist; GOLD, Global initiative for Obstructive Lung Disease; $\mathrm{FEV}_{\mathrm{I}}$, forced expiratory volume in I second. 
for COPD treatment are unclear regarding treatment changes. Accordingly, clinicians used their own experience and knowledge of the disease to make these decisions. Thus, treatment changes may be influenced by hospital practices, known as a "clustering effect". This clustering effect indicates that patients with similar characteristics may experience different processes of care, depending on the hospital in which they are evaluated. ${ }^{24}$ These factors include otherwise unevaluated aspects that might influence clinical care, including local guidelines and policies, pricing and reimbursement policies, practices of individual clinicians, center policies, cultural practices that may influence treatment decisions, or clinical characteristics that may be observed within geographical clusters due to factors that are common to the local population, such as sociodemographic factors, family and social support networks, cultural beliefs and attitudes, and environmental susceptibilities. An awareness of this situation is especially important in databases with a hierarchical structure like ours. In these cases, a multilevel multivariate analysis helps to evaluate and quantify the importance of the hospital cluster effect. ${ }^{25}$ In our case, we did not have the power to perform this multilevel analysis, but we were able to identify some centers with a different practice in stepping up or discontinuing ICS.

Current guidelines recommend directing therapy based on three main variables: lung function, symptoms, and exacerbations. According to our results, these variables were indeed factors in stepping up. Exacerbations were the main drive in stepping up treatment, as were symptoms, which was expected. Both symptoms and exacerbations have been associated with clinically relevant outcomes. Interestingly, spirometric severity or the classification types were not associated with a step-up in treatment. This result suggests that pulmonologists guide their step-up decisions based on clinical grounds. This finding continues the debate as to whether spirometry should be used for the follow-up of patients or if clinical outcomes alone should guide therapy. ${ }^{26}$

Of note, clinicians did not use symptoms for stepping down. Instead, they used $\mathrm{FEV}_{1}$ rather than symptoms, and previous treatments with long-term antibiotics or ICS were the key determinants to step-down treatment according to our data. Therefore, it appears that of the three GOLD axes (lung function, exacerbations, and symptoms), pulmonologists use symptoms and exacerbation to step-up and lung function to step-down. Nonetheless, caution should be exercised when interpreting the associations identified in the current study. The significance of $\mathrm{FEV}_{1}$ is quite intuitive. The higher the $\mathrm{FEV}_{1}$, the greater the likelihood of step-down treatment.
However, the observation that symptoms or exacerbations were not retained into the multivariate model is surprising. One possible explanation is the overprescription reported in patients with COPD for certain $\mathrm{FEV}_{1}$ values. ${ }^{18}$ In this scenario, clinicians may decide to step-down intensive treatments in stable patients who receive too many medications for a given level of lung function impairment. The use of ICS as a predictor of step-down is also expected because the most common step-down strategy was based on ICS discontinuation. The use of preventive antibiotic therapy is part of the controversy. GOLD recommendations indicate that the use of antibiotics is currently not indicated except for treating infectious exacerbations of COPD and other bacterial infections. ${ }^{3}$ However, the Spanish guidelines (GesEPOC) recommend long-term antibiotics for a selected group of patients with frequent infectious exacerbations. ${ }^{27}$ This discrepancy in the recommendations leads to confusion. As a result, doctors also make decisions based on their personal beliefs and clinical experience. The association of antibiotics with step-down can be explained by the fact that the antibiotic itself has been withdrawn (which could be in turn due to a lack of efficacy or a previous prescription deemed to be inappropriate by the current physician). However, only two of these cases were observed in our study. Alternatively, the antibiotic could have been clinically useful, leading to discontinuation of ICS and/or another medication. In our dataset, only changes in medication were recorded. Unfortunately, the reasons that motivated such modifications were not collected.

ICS discontinuation warrants a particular comment. One expected finding was that the number of previous exacerbations would be a key finding to discontinue ICS. However, this expectation was not confirmed. The majority of variables associated with ICS discontinuation were protective in nature. Accordingly, the more severe the disease, as indicated by either GOLD patient type or lung function impairment, the less likely ICS was to be discontinued. Additionally, the presence of asthma-like symptoms was also associated with not discontinuing ICS. The only positive association was receiving two long-acting bronchodilators, which may have helped doctors feel more confident about discontinuing ICS. This finding is interesting because current literature suggests that ICS can be discontinued not only in certain cases in clinical trials with double bronchodilation ${ }^{11}$ but also in actual clinical practice with different therapeutic schemes. ${ }^{28}$ As a consequence of this dilemma, the Spanish National Respiratory Society released a consensus document providing information on the correct use of ICS in COPD. ${ }^{29}$ 
Interestingly, fixed combinations of long-acting bronchodilators $(\mathrm{LAMA}+\mathrm{LABA})$ in one single inhaler were not available in our country when the audit was performed. We therefore assessed the combination of two bronchodilators in distinct inhalers. We acknowledge that the use of fixed combinations of long-acting bronchodilators (LAMA+LABA) in one single inhaler could have modified the prescription patterns. However, the clinical efficacy of two long-acting bronchodilators is identical regardless of their administration in one or two inhalers. ${ }^{30}$ In addition, severe patients were already on ICS-LABA FDCs,${ }^{18}$ making the expected impact of this variable likely to be noninfluential.

The evaluation of clinical phenotypes in the management of COPD is a novel approach initially proposed in the Spanish COPD guidelines (GesEPOC) ${ }^{4}$ and subsequently adopted by other countries. ${ }^{31}$ In this context, it will be important to explore how these phenotypes can be used in clinical practice and how they may influence treatment selection. In our study, the results of bivariate analyses demonstrated that certain GesEPOC phenotypes were associated with step-down or ICS discontinuation, but not with step-up. However, such differences were not retained in the multivariate model. Interestingly, specific phenotyperelated features (eg, asthma-like symptoms or number of exacerbations) were statistically significant in multivariate analysis. These results suggest that physicians' prescriptions are mainly based on single independent features, rather than complex phenotypes.

\section{Conclusion}

In conclusion, this study analyzed the prescribed treatments at a routine follow-up visit of COPD, indicating the determinants of treatment change. The results indicate that the majority of doctors did not change the treatment regimen, and for those patients whose treatments were changed, there were a number of influential factors. In particular, pulmonologists seem to use symptoms and exacerbations to step-up and lung function to step-down. Further trials are needed to evaluate whether these therapeutic changes impact clinically relevant outcomes at follow-up. Currently, a degree of uncertainty remains regarding treatment adaptation over time as the disease progresses. Future guidelines should attempt to advocate for therapeutic regimens to enable a more individualized treatment for COPD.

\section{Acknowledgments}

This study was financially supported by an unrestricted grant from Laboratorios Menarini, SA (Barcelona, Spain). The authors are grateful to all of the project researchers for their time and dedication to this project.

\section{Disclosure}

The authors report no conflicts of interest in this work.

\section{References}

1. Agusti A, Calverley PM, Celli B, et al. Characterisation of COPD heterogeneity in the ECLIPSE cohort. Respir Res. 2010; $11: 122$.

2. Kessler R, Partridge MR, Miravitlles M, et al. Symptom variability in patients with severe COPD: a pan-European cross-sectional study. Eur Respir J. 2011;37(2):264-272.

3. Vestbo J, Hurd SS, Agusti AG, et al. Global strategy for the diagnosis, management, and prevention of chronic obstructive pulmonary disease: GOLD executive summary. Am J Respir Crit Care Med. 2013; 187(4):347-365.

4. Miravitlles M. What was the impact of the Spanish COPD guidelines (GesEPOC) and how can they be improved? Arch Bronconeumol. 2016; 52(1):1-2.

5. Lopez-Campos JL, Bustamante V, Munoz X, Barreiro E. Moving towards patient-centered medicine for COPD management: multidimensional approaches versus phenotype-based medicine - a critical view. COPD. 2014;11(5):591-602.

6. Soriano JB, Rodriguez-Roisin R. Chronic obstructive pulmonary disease overview: epidemiology, risk factors, and clinical presentation. Proc Am Thorac Soc. 2011;8(4):363-367.

7. Pierson DJ. Clinical practice guidelines for chronic obstructive pulmonary disease: a review and comparison of current resources. Respir Care. 2006;51(3):277-288.

8. Overington JD, Huang YC, Abramson MJ, et al. Implementing clinical guidelines for chronic obstructive pulmonary disease: barriers and solutions. J Thorac Dis. 2014;6(11):1586-1596.

9. Diette GB, Dalal AA, D’Souza AO, Lunacsek OE, Nagar SP. Treatment patterns of chronic obstructive pulmonary disease in employed adults in the United States. Int J Chron Obstruct Pulmon Dis. 2015;10: 415-422.

10. Nardini S, Camiciottoli G, Locicero S, et al. COPD: maximization of bronchodilation. Multidiscip Respir Med. 2014;9(1):50.

11. Rodriguez Roisin R, Arismendi E. Inhaled corticosteroids withdrawal in severe patients with chronic obstructive pulmonary disease: a wisdom decision? Arch Bronconeumol. 2015;51(2):57-58.

12. Wilson R, Sethi S, Anzueto A, Miravitlles M. Antibiotics for treatment and prevention of exacerbations of chronic obstructive pulmonary disease. J Infect. 2013;67(6):497-515.

13. Martinez FJ, Calverley PM, Goehring UM, Brose M, Fabbri LM, Rabe KF. Effect of roflumilast on exacerbations in patients with severe chronic obstructive pulmonary disease uncontrolled by combination therapy (REACT): a multicentre randomised controlled trial. Lancet. 2015;385(9971):857-866.

14. Osthoff M, Jenkins C, Leuppi J. Chronic obstructive pulmonary diseasea treatable disease. Swiss Med Wkly. 2013;143:w13777.

15. Lopez-Campos JL, Abad Arranz M, Calero Acuna C, et al. Clinical audits in outpatient clinics for chronic obstructive pulmonary disease: methodological considerations and workflow. PloS One. 2015;10(11): e0141856.

16. Pozo-Rodriguez F, Alvarez CJ, Castro-Acosta A, et al. Auditoría clínica de los pacientes hospitalizados por exacerbación de EPOC en España (estudio AUDIPOC): método y organización del trabajo [Clinical audit of patients admitted to hospital in Spain due to exacerbation of COPD (AUDIPOC study): method and organisation]. Arch Bronconeumol. 2010;46(7):349-357. Spanish.

17. Hartl S, Lopez-Campos JL, Pozo-Rodriguez F, et al. Risk of death and readmission of hospital-admitted COPD exacerbations: European COPD Audit. Eur Respir J. 2016;47(1):113-121. 
18. Price D, West D, Brusselle G, et al. Management of COPD in the UK primary-care setting: an analysis of real-life prescribing patterns. Int J Chron Obstruct Pulmon Dis. 2014;9:889-904.

19. Ford ES, Mannino DM, Wheaton AG, et al. Trends in the use, sociodemographic correlates, and undertreatment of prescription medications for chronic obstructive pulmonary disease among adults with chronic obstructive pulmonary disease in the United States from 1999 to 2010. PloS One. 2014;9(4):e95305.

20. Izquierdo JL, Martin A, de Lucas P, Rodriguez-Gonzalez-Moro JM, Almonacid C, Paravisini A. Misdiagnosis of patients receiving inhaled therapies in primary care. Int J Chron Obstruct Pulmon Dis. 2010;5: 241-249.

21. Flottorp SA, Jamtvedt G, Gibis B, McKee M. Using Audit and Feedback to Health Professionals to Improve the Quality and Safety of Health Care. Copenhagen: World Health Organization; 2010.

22. Lopez-Campos JL, Asensio-Cruz MI, Castro-Acosta A, et al. Results from an audit feedback strategy for chronic obstructive pulmonary disease in-hospital care: a joint analysis from the AUDIPOC and European COPD audit studies. PloS One. 2014;9(10):e110394.

23. Nardini S, Cicchitto G, De Benedetto F, et al. Audit on the appropriateness of integrated COPD management: the "ALT-BPCO" project. Multidiscip Respir Med. 2014;9(1):40.

24. Glaser D, Hastings RH. An introduction to multilevel modeling for anesthesiologists. Anesth Analg. 2011;113(4):877-887.

25. Merlo J. Multilevel analytical approaches in social epidemiology: measures of health variation compared with traditional measures of association. J Epidemiol Community Health. 2003;57(8):550-552.
26. Woodruff PG, Agusti A, Roche N, Singh D, Martinez FJ. Current concepts in targeting chronic obstructive pulmonary disease pharmacotherapy: making progress towards personalised management. Lancet. 2015;385(9979):1789-1798.

27. Miravitlles M, Soler-Cataluna JJ, Calle M, et al. Spanish COPD Guidelines (GesEPOC): pharmacological treatment of stable COPD. Spanish Society of Pulmonology and Thoracic Surgery. Arch Bronconeumol. 2012;48(7):247-257.

28. Rossi A, Guerriero M, Corrado A, Group OAS. Withdrawal of inhaled corticosteroids can be safe in COPD patients at low risk of exacerbation: a real-life study on the appropriateness of treatment in moderate COPD patients (OPTIMO). Respir Res. 2014;15:77.

29. Alcazar Navarrete B, Casanova C, Miravitlles M, et al. "Correct use of inhaled corticosteroids in chronic obstructive pulmonary disease": a consensus document. Arch Bronconeumol. 2015;51(4):193-198.

30. Dahl R, Jadayel D, Alagappan VK, Chen H, Banerji D. Efficacy and safety of QVA149 compared to the concurrent administration of its monocomponents indacaterol and glycopyrronium: the BEACON study. Int J Chron Obstruct Pulmon Dis. 2013;8:501-508.

31. Miravitlles M, Vogelmeier C, Roche N, et al. A review of national guidelines for management of COPD in Europe. Eur Respir J. 2016; 47(2):625-637.
International Journal of COPD

\section{Publish your work in this journal}

The International Journal of COPD is an international, peer-reviewed journal of therapeutics and pharmacology focusing on concise rapid reporting of clinical studies and reviews in COPD. Special focus is given to the pathophysiological processes underlying the disease, intervention programs, patient focused education, and self management protocols.

\section{Dovepress}

This journal is indexed on PubMed Central, MedLine and CAS. The manuscript management system is completely online and includes a very quick and fair peer-review system, which is all easy to use. Visit http://www.dovepress.com/testimonials.php to read real quotes from published authors. 